SCIENTIFIC LETTER

\title{
Oral anticoagulation in patients aged 75 years or older with chronic non-valvar atrial fibrillation: effectiveness and safety in daily clinical practice
}

\author{
M Ruiz Ortiz, E Romo Peñas, M F Franco Zapata, D Mesa Rubio, M Anguita Sánchez, A López \\ Granados, J M Arizón del Prado, F Vallés Belsué
}

Heart 2005;91:1225-1226. doi: 10.1136/hrt.2004.050831

\begin{abstract}
A Ithough American College of Cardiology/American Heart Association/European Society of Cardiology clinical practice guidelines ${ }^{1}$ recommend oral anticoagulation for patients aged 75 years or older with nonvalvar atrial fibrillation (NVAF) and without contraindication to this treatment, underutilisation has often been reported. ${ }^{2}$ Moreover, this population has been underrepresented in clinical trials - the only one that included a high proportion of elderly patients ${ }^{3}$ had equivocal results: a reduction of embolic events, but an increase in severe bleeding, especially intracranial, that offset the benefit obtained. We planned a prospective observational cohort study with the following objectives: to describe cardioembolic risk factors and contraindications to anticoagulants in our population of elderly patients; to manage oral anticoagulation prescription in this group by trying to follow the guidelines; and to evaluate whether oral anticoagulation in our outpatient cardiology clinic is effective and safe to lessen thromboembolic events in elderly patients with NVAF. We have previously reported the usefulness of a prospective protocol in this setting to increase oral anticoagulation prescription. ${ }^{4}$ This work describes basal and follow up data of the subgroup of patients aged 75 years and older. This protocol received the usual ethical approval for observational studies in our institution, and all patients gave informed consent. Patient selection criteria and methods have been described. ${ }^{4}$
\end{abstract}

\section{METHODS}

From l February 2000 to l February 2002, 279 consecutive patients with chronic NVAF who were not candidates for cardioversion attended our clinic. All of them agreed to participate in the study. Mean (SD) age was 80 (4) years and 96 (34\%) were men. NVAF was most often related to hypertension ( 172 of $279(62 \%)$ ), followed by the absence of structural heart disease $(66(24 \%))$, coronary heart disease (25 $(9 \%))$, cardiomyopathy $(12(4 \%))$, and other heart diseases $(4(1 \%))$. Observed cardioembolic risk factors were hypertension (197 of $279(71 \%))$, diabetes (64 (23\%)), congestive heart failure (51 $(18 \%))$, a prior cardioembolic event $(39(14 \%))$, atrial enlargement $(31(11 \%))$, coronary heart disease $(25(9 \%))$, and left ventricular dysfunction (18 $(7 \%))$. Only 43 patients had no other risk factors (15\%), whereas 123 had one additional cardioembolic risk factor other than age $75(44 \%)$ and 66 had two (24\%). Seventy patients $(25 \%)$ had at least one contraindication to oral anticoagulation: the most common was a high risk of significant treatment non-compliance (50 of 70 (71\%)), followed by digestive disease (6 (9\%)), uncontrolled hypertension $(4(6 \%))$, a high probability of severe trauma (4 $(6 \%))$, recent major bleeding $(3(4 \%))$, and anaemia (3 $(4 \%))$.

Patients with contraindications to oral anticoagulation received aspirin or antiplatelets. Those with at least one additional cardioembolic risk factor (apart from age) and no contraindication to oral anticoagulation were offered anticoagulation. Lastly, treatment of patients without contraindications and with only advanced age as a cardioembolic risk factor was left to the responsible physician to decide. Estimated risks and benefits of treatment were fully discussed with patients and their families, and they were informed of possible alternatives. Every patient participated in the decision making process and enough time was spent in the discussion to avoid incomplete or inadequate information. Doses of medication were adjusted by experienced haematologists, who did not know about the study, with a target international normalised ratio (INR) of 2 to 3 .

Subgroups were compared by Student's $t$ test for quantitative data and $\chi^{2}$ or Fisher's exact test for qualitative data. Event-free survival was analysed with the Kaplan-Meier actuarial method, with the log rank test used for subgroup comparisons. SPSS software version 8.0 (SPSS Inc, Chicago, Illinois, USA) was used for statistical analysis.

\section{RESULTS}

Of 209 patients without contraindications, 17 declined anticoagulant treatment and finally 163 received anticoagulants (58\% of the series). Anticoagulated patients were younger than unanticoagulated patients (79 (3) $v$ 81 (4) years, $\mathrm{p}<0.001)$ and had a higher prevalence of hypertension $(76 \% \vee 63 \%, \mathrm{p}=0.018)$, diabetes $(29 \% \vee 14 \%$, $\mathrm{p}=0.002)$, previous embolism $(19 \% \vee 7 \%, \mathrm{p}=0.004)$, and coronary heart disease $(14 \% v 3 \%, \mathrm{p}=0.002)$, a greater number of cardioembolic risk factors $(2.8$ (1.1) $v 2.1(0.9)$, $\mathrm{p}<0.001$ ), and a lower prevalence of lone atrial fibrillation ( $17 \%$ v 33\%, $\mathrm{p}=0.003$ ) than non-anticoagulated patients. Most unanticoagulated patients received antiplatelets ( 108 of $116(93 \%))$, mainly aspirin (103 of $108(95 \%)$ ).

After a mean (SD) follow up of 20 (14) months, with data for 270 patients, 160 anticoagulated (268 patient years) and 110 non-anticoagulated (182 patient years), events in both groups were as follows: transient ischaemic attacks $0(0 \%) \vee 5$ $(2.74 \%)$, non-fatal strokes $2(0.75 \%) \vee 7(3.85 \%)$, fatal strokes $0(0 \%) v 3(1.65 \%)$, peripheral embolism $0(0 \%) v 1(0.55 \%)$, non-fatal severe bleeding $6(2.24 \%) \vee 2(1.1 \%)$, fatal severe bleeding $\mathrm{l}(0.37 \%) v 0(0 \%)$, and deaths from other causes 8 $(2.99 \%) v 12(6.59 \%)$. Despite a worse cardioembolic profile, anticoagulated patients had a lower rate of annual embolic events (transient ischaemic attack, stroke, or peripheral embolism) $(0.75 \% \vee 8.79 \%, \mathrm{p}<0.001)$ and total mortality $(3.36 \%$ v $8.24 \%, p=0.023)$, without significant differences in severe bleeding rate $(2.61 \% \vee 1.10 \%, p=0.25)$. INRs at admission for the anticoagulated patients with stroke were 1.7 and 1.9. Most non-fatal severe bleeding events were of gastrointestinal origin in both groups (five of eight $(63 \%)$ ), but two anticoagulated patients had a non-fatal intracranial 


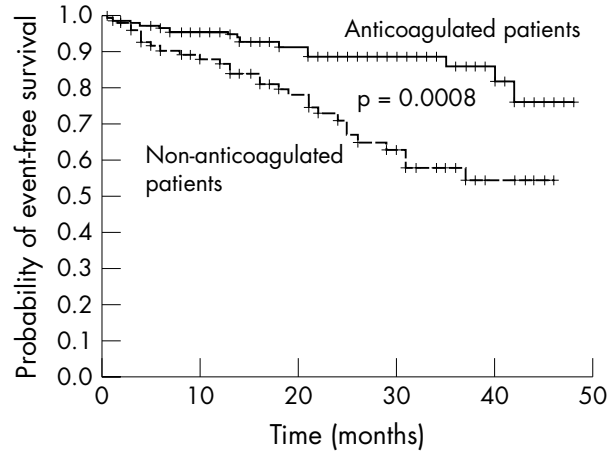

Figure 1 Probability of survival free from embolism or severe bleeding by treatment group.

haemorrhage. The only fatal bleeding event was a digestive haemorrhage in an anticoagulated patient. Mean INR at admission for the anticoagulated patients with severe bleeding was $4.4(2.1)$ (range 2.7-8.8). Survival free from embolism or haemorrhage was significantly better in anticoagulated patients (fig 1).

\section{DISCUSSION}

Our results confirm that patients 75 years or older with NVAF have a high prevalence of additional cardioembolic risk factors other than age: most patients had one or two risk factors, the most common of which were hypertension and diabetes. Some studies ${ }^{5}$ conclude that, in the context of shared decision making, taking account of an elderly patient's preference would lead to fewer prescriptions for oral anticoagulation than under published guidelines. In our study, although patients' preferences were always taken into account, we did not use a formal decision analysis because its efficacy and safety have not been proved conclusively. We tried to follow guidelines, achieving a 58\% rate of anticoagulation prescription, which is among the highest rates reported in this population in observational studies. Follow up data confirm that oral anticoagulation is effective and safe in elderly patients with NVAF.

\section{Authors' affiliations}

M Ruiz Ortiz, E Romo Peñas, M Franco Zapata, D Mesa Rubio, M Anguita Sánchez, A López Granados, J M Arizón del Prado, F Vallés Belsué, Department of Cardiology, Hospital Reina Sofía, Cordoba, Spain

This study has received a grant from the Mapfre Medicine Foundation.

Correspondence to: DrM Ruiz Ortiz, C/Huerta del Recuero 1, portal 732, 14011 Cordoba, Spain; mruor@supercable.es

Accepted 14 December 2004

\section{REFERENCES}

1 Fuster V, Rydén LE, Asinger RW, et al. ACC/AHA/ESC guidelines for the management of patients with atrial fibrillation. J Am Coll Cardiol 2001;38:1231-66.

2 Ezekowitz MD, Falk RH. The increasing need for anticoagulant therapy to prevent stroke in patients with atrial fibrillation. Mayo Clin Proc 2004;79:904-13.

3 Stroke Prevention in Atrial Fibrillation Investigators. Warfarin versus aspirin for prevention of thromboembolism in atrial fibrillation. Stroke prevention in atrial fibrillation II study. Lancet 1994;343:687-91.

4 Ruiz Ortiz M, Romo Peñas E, Franco Zapata M, et al. A prospective protocol increases oral anticoagulant prescription in patients with chronic nonvalvular atrial fibrillation. Rev Esp Cardiol 2003;56:971-7.

5 Protheroe J, Fahey T, Montgomery AA, et al. The impact of patients' preferences on the treatment of atrial fibrillation: observational study of patient based decision analysis. BMJ 2000;320:1380-4

\section{IMAGES IN CARDIOLOGY}

\section{Coronary angiography in a patient with pulmonary alveolar microlithiasis}

A 52 year old woman with severe chronic respiratory insufficiency caused by pulmonary alveolar microlithiasis was admitted for invasive evaluation, including right and left heart catheterisation, before planned lung transplantation. Right heart catheterisation demonstrated high grade secondary pulmonary hypertension (mean pulmonary artery pressure $51 \mathrm{~mm} \mathrm{Hg}$ ). Coronary angiography did not reveal evidence of atherosclerotic coronary artery disease. However, even with high resolution digital flat panel fluoroscopy, image quality was poor because of diffuse, nodular ("sandstorm-like") calcifications in both lungs, which are characteristic for pulmonary alveolar microlithiasis.

Pulmonary alveolar microlithiasis is a rare disease of unknown pathogenesis, characterised by widespread intra-alveolar calcium deposits (calci spherites) in the absence of any known disorder of calcium metabolism. It usually occurs in a sporadic form, but also an autosomal recessive form has been described. Patients usually remain asymptomatic for many years. At adult age, however, patients commonly show progressive deterioration of pulmonary function with restrictive pattern, ultimately resulting in respiratory failure associated with cor pulmonale. Lung transplantation is the only effective treatment
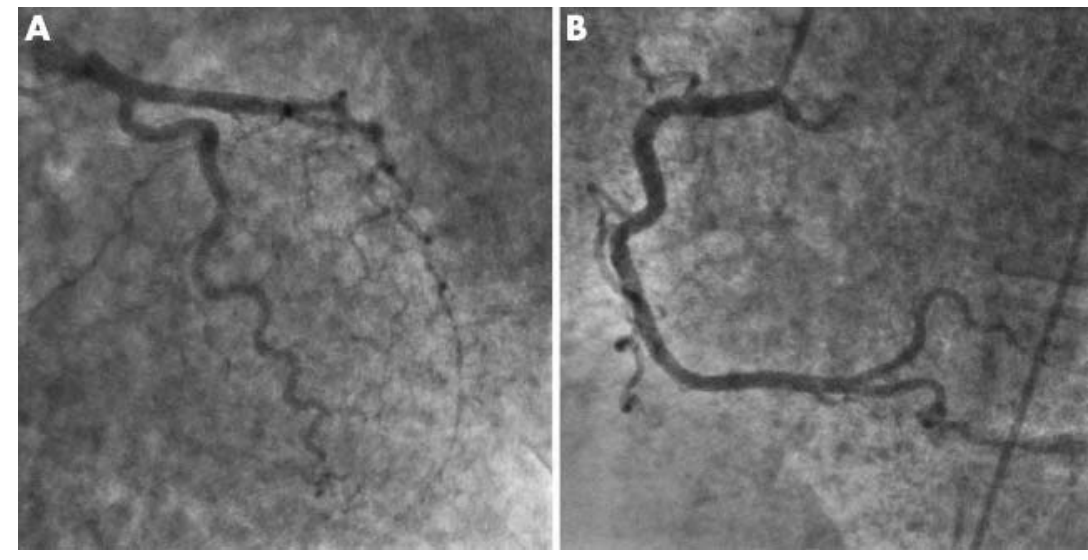

Digital flat panel coronary angiography of the left anterior artery (panel A) and the right coronary artery (panel B). Note the characteristic, diffuse calcifications in both lungs caused by pulmonary alveolar microlithiasis, significantly impairing image quality.
H Kälsch

H Eggebrecht

$R$ Erbel

h.kaelsch@gmx.de 